\title{
Analysis of Go-Back-N ARQ in Block Fading Channels
}

\author{
Kamtorn Ausavapattanakun and Aria Nosratinia, Senior Member, IEEE
}

\begin{abstract}
This work analyzes the throughput of Go-Back-N (GBN) in block fading, a model frequently used for slow fading wireless channels. We devise hidden Markov models and block transition probabilities for the block fading channel, allowing us to calculate the throughput of GBN with reliable feedback, as well as unreliable feedback. The advantages of this approach include generality: it applies to two-state as well as multi-state models for forward and reverse channels. Also, the results for both reliable and unreliable feedback are expressed in terms of probability matrices, which can be used as convenient building blocks in the analysis and simulation of larger systems. Simulations verify our analysis.
\end{abstract}

Index Terms-ARQ, block fading, Go-Back-N protocol, throughput.

\section{INTRODUCTION}

G O-Back-N (GBN) protocol is one of three basic ARQ schemes, which are widely used in packet communication systems. In this paper, we compute the throughput of GBN in fading wireless channels.

A brief summary of past works related to this area is as follows. Several studies [1]-[4] have modeled the wireless channel as a finite-state Markov process. The performance of GBN under Markov errors has been reported in [5]-[8]. These works either assume error-free feedback or use a twostate Markov model in both forward and backward channels. Part of our contribution is to relax both these assumptions. Turin [9] calculates the throughput of GBN under a hidden Markov model (HMM), by transforming the parameters of the HMM in a way to make the analysis tractable. He also assumes bit-reversal errors (instead of erasures) in the feedback link.

This work studies GBN under block fading, a popular model for slow fading channels [10]. The key challenge for analysis under block fading is that the instantaneous channel condition does not by itself represent the history of the channel. One must also know the relative time index within the fading block. Thus simple models, such as a two-state time-invariant Markov model, cannot be used. To make the analysis tractable, in the case where the feedback link is reliable, we develop a multi-state, cyclo-stationary hidden Markov model for the channel, and use the method of Turin [9] to analyze it.

When the feedback channel is unreliable, one must construct a composite channel for the purpose of analysis. To manage the complications arising in this task, Turin [9] assumed bitreversal errors for the HMM channel, thus an error in the

Manuscript received January 23, 2006; revised October 26, 2006; accepted October 27, 2006. The associate editor coordinating the review of this letter and approving it for publication was R. Fantacci.

The authors are with the Department of Electrical Engineering, the University of Texas at Dallas, Richardson, TX 75080 USA (e-mail: \{kxa025000, aria\}@utdallas.edu).

Digital Object Identifier 10.1109/TWC.2007.06062. feedback link would convert ACK to NACK and vice versa. This assumption simplifies the analysis, but unfortunately it departs from common practice. Modern packet communication systems use CRC to detect any errors, and erroneous packets are discarded (erasure). More importantly, the bit-reversal can lead to problems in the GBN protocol itself: In GBN, the receiver processes frames in a certain order. If a frame is erroneous, the receiver will send a NACK and reject all subsequent frames until the missing frame is received. Now if the NACK turns to ACK by the time it reaches the transmitter, the corresponding frame will never be re-transmitted and the protocol will go into deadlock. Also, because the transmitter has received an ACK, this deadlock cannot be resolved through a timeout.

We are able to address erasure errors on both the forward and reverse link by using block-transition probabilities. To prevent deadlock when a NACK is lost in transition, we use a time-out mechanism as in [7], [8]. Simulations confirm the validity of our result in a variety of scenarios.

To summarize, in this work we (a) characterize block fading via a nonstationary hidden Markov model, (b) include erasure errors in both directions, and (c) analyze GBN with timeouts under this scenario, which is more realistic than the previous works. A key contribution of this work is to unify all these factors under one umbrella and provide analysis for GBN in block fading channels, which was not available previously.

\section{Block FAding ANd Hidden MARKov Models}

We denote by $Y_{t}$ the discrete-valued random variable representing channel quality at time $t$, taking values from the set $\mathcal{Y}=\{1,2, \ldots, K\}$. At any given time $t$, the observation of this channel (error or no error) is characterized by a Bernoulli random variable $X_{t}$ taking values over $\mathcal{X}=\{0,1\}$. The probability of error is defined as $\varepsilon_{j}=\operatorname{Pr}\left[X_{t}=1 \mid Y_{t}=j\right]$. Error probability is thus a function of channel quality. If $Y_{t}$ is a Markov chain (with states $\mathcal{Y}$ and transition probability matrix $\mathbf{P})$, then $X_{t}$ will be a hidden Markov chain characterized by $\{\mathcal{Y}, \mathcal{X}, \mathbf{P}, \varepsilon\}$.

The hidden Markov model can describe a number of physical channels, for example the fading wireless channel. However, for slow fading, often the block fading model is used, where the channel condition does not transition every time, but only once every $N$ time intervals. Thus, the transition probabilities of the channel are not time-invariant.

To cast the problem once again in the framework of hidden Markov processes, we need to expand the state space. In particular, let the time index inside a block be represented by the index $n$ taking values over $\mathcal{N}=\{1, \ldots, N\}$. Then the expanded state space is defined as the Cartesian product 
$s=(n, j)$ taking values over $\mathcal{S}=\mathcal{N} \times \mathcal{Y}$. The transition probabilities between the states are as follows. For $i, j \in \mathcal{Y}$ and $n=1,2, \cdots N-1$

$$
\begin{aligned}
\operatorname{Pr}\left[S_{t}=(m, i) \mid S_{t-1}=(n, j)\right] \\
\quad= \begin{cases}1, & \text { if } m=n+1 \text { and } i=j \\
0, & \text { otherwise }\end{cases}
\end{aligned}
$$

For $i, j \in \mathcal{Y}$ and $n=N$

$$
\begin{aligned}
\operatorname{Pr}\left[S_{t}=(m, i) \mid\right. & \left.S_{t-1}=(n, j)\right] \\
& = \begin{cases}\operatorname{Pr}\left[Y_{t}=i \mid Y_{t-1}=j\right], & \text { if } m=1 \\
0, & \text { otherwise }\end{cases}
\end{aligned}
$$

Then $S_{t}$ is a Markov chain whose state transition matrix we denote with $\mathbf{M}$. To write the state transition matrix, we need a linear ordering of the states. The ordering does not affect the outcome of analysis, therefore we assume an arbitrary ordering and, with an abuse of notation, show it with the same symbol $s=1,2, \ldots, N K$. In each state $s$ the channel will have an error probability that we denote with $\varepsilon_{s}$, and the vector of all such probabilities is denoted with $\varepsilon$.

For our examples we concentrate on the simple case where $\mathcal{Y}$ has only two values, representing the good and bad fading situations. The state transition diagram is shown in Fig. 1. For clarity the states are labeled $\left\{G_{1}, B_{1}, \ldots, G_{N}, B_{N}\right\}$, where $G_{i}$ and $B_{i}$ represent the channel in a good and bad fading state, respectively, and the relative time index within fading block is $i$.

Thus we have arrived at a hidden Markov model described by the quartet $\{\mathcal{S}, \mathcal{X}, \mathbf{M}, \varepsilon\}$, where $\varepsilon$ is a vector of error probabilities in all (hidden) states. For the purposes of analysis, however, it is more convenient to use an alternative representation of the Markov chain that combines the last two parameters into one (set of parameters), following the formulation of Turin. In the special case of Bernoulli observations, the formulation is as follows. Let $\mathbf{A}_{1}=\operatorname{diag}\{\boldsymbol{\varepsilon}\}$ and $\mathbf{A}_{0}=\mathbf{I}-\mathbf{A}_{1}$. Define $\mathbf{M}_{i}=\mathbf{M} \mathbf{A}_{i}$ for $i=0,1$. Then the HMM is fully specified with $\left\{\mathcal{S}, \mathcal{X}, \mathbf{M}_{0}, \mathbf{M}_{1}\right\}$ [9]. Note that $\mathbf{M}=\mathbf{M}_{0}+\mathbf{M}_{1}$. A similar construction is possible for larger observation alphabets, leading to more $\mathbf{M}_{i}$ matrices.

\section{GBN ThroughPUT ANALYSIS}

We assume the source always has data to transmit, all frames have the same length, and it takes one time slot to transmit a frame. The round trip time is $k$, i.e. between the time a frame is transmitted and its acknowledgment is received, $k-1$ more frames are sent. Thus, if a NACK for the frame transmitted at time $t$ is received, the frame will be retransmitted at time $t+k$.

\section{A. Reliable Feedback}

Using the setup constructed in the last section, the throughput of GBN with reliable feedback can be calculated for any set of channel qualities and any block length, in a manner following [9]. For example, consider block fading of length

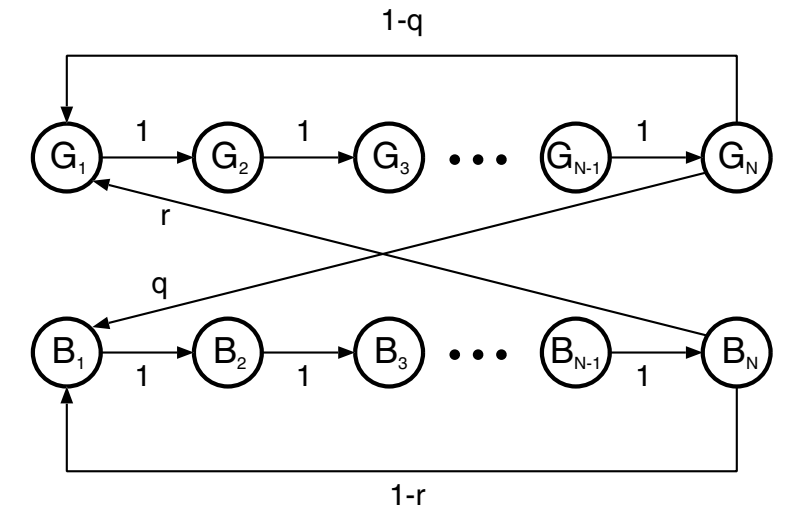

Fig. 1. The state transition diagrams of block fading with two channel qualities.

$N=3$ and two channel qualities (good and bad). Once every 3 time intervals, the channel quality is updated according to the transition matrix

$$
\mathbf{P}=\left[\begin{array}{cc}
1-q & q \\
r & 1-r
\end{array}\right]
$$

where the first row and column corresponds to the good channel condition, and the second row and column to the bad channel condition. Now, for a Markov system that transitions every time interval, using (1) and (2), the state transition probability matrix will be

$$
\begin{aligned}
\mathbf{M} & =\left[\begin{array}{cccccc}
0 & 0 & 1 & 0 & 0 & 0 \\
0 & 0 & 0 & 1 & 0 & 0 \\
0 & 0 & 0 & 0 & 1 & 0 \\
0 & 0 & 0 & 0 & 0 & 1 \\
1-q & q & 0 & 0 & 0 & 0 \\
r & 1-r & 0 & 0 & 0 & 0
\end{array}\right] \\
& =\left[\begin{array}{cc}
\mathbf{0}_{\mathbf{1}} & \mathbf{I} \\
\mathbf{P} & \mathbf{0}_{\mathbf{2}}
\end{array}\right]
\end{aligned}
$$

The error probability is equal to $\varepsilon_{G}$ and $\varepsilon_{B}$ for the states corresponding to the good and bad channel qualities, respectively. The vector of error probabilities is $\varepsilon=$ $\left[\varepsilon_{G} \varepsilon_{B} \varepsilon_{G} \varepsilon_{B} \varepsilon_{G} \varepsilon_{B}\right]$. The diagonal matrices of the state error and success probabilities are $\mathbf{A}_{1}=\operatorname{diag}\{\boldsymbol{\varepsilon}\}$ and $\mathbf{A}_{0}=\mathbf{I}-\mathbf{A}_{1}$

Finally we have $\left\{\mathcal{S}, \mathcal{X}, \mathbf{M}_{0}, \mathbf{M}_{1}\right\}$ as a HMM channel where $\mathbf{M}_{0}=\mathbf{M} \mathbf{A}_{0}$ and $\mathbf{M}_{1}=\mathbf{M} \mathbf{A}_{1}$. Notice that the state transition probability $\mathbf{M}$ is periodic with period $N$ so the limiting state probabilities do not exist [11]. However, the stationary probabilities can be found by solving the following system of equations.

$$
\pi \mathbf{M}=\pi \quad \text { and } \quad \pi \mathbf{1}=1
$$

where 1 is a column vector of ones. The channel is error free, therefore we have

$$
\eta=\pi\left[\mathbf{I}-\mathbf{M}_{1} \mathbf{Q}(k)\right]^{-1} \mathbf{M}_{0} \mathbf{1}
$$

where $k$ is the round trip time, $\mathbf{I}$ is an identity matrix and $\mathbf{Q}(k)=\sum_{i=0}^{k-2} \mathbf{M}^{i}$. 


\section{B. Noisy Feedback}

As described in [12], if forward and backward channels are independent and subject to hidden Markov errors, the composite channel can also be described by a HMM model. Let $\left\{\mathcal{S}^{(f)}, \mathcal{X}^{(f)}, \mathbf{M}_{0}^{(f)}, \mathbf{M}_{1}^{(f)}\right\}$ and $\left\{\mathcal{S}^{(b)}, \mathcal{X}^{(b)}, \mathbf{M}_{0}^{(b)}, \mathbf{M}_{1}^{(b)}\right\}$ be the forward and backward channels respectively. Then, the composite channel is characterized by $\left\{\mathcal{S}, \mathcal{X}, \mathbf{M}_{00}, \mathbf{M}_{01}, \mathbf{M}_{10}, \mathbf{M}_{11}\right\}$ where $\mathcal{S}=\mathcal{S}^{(f)} \times \mathcal{S}^{(b)}$, $\mathcal{X}=\mathcal{X}^{(f)} \times \mathcal{X}^{(b)}$, and each of the observation probability matrices is described by a Kronecker product, i.e, $\mathbf{M}_{i j}=$ $\mathbf{M}_{i}^{(f)} \otimes \mathbf{M}_{j}^{(b)}$ for $i, j=0,1$. In this context, $X_{t}=00$ means both channels are good while $X_{t}=01$ means the backward channel is erroneous. Note that $\mathbf{M}=\mathbf{M}_{00}+\mathbf{M}_{01}+\mathbf{M}_{10}+$ $\mathbf{M}_{11}$.

As mentioned earlier, [9] assumed bit-reversal errors in the feedback channel, which leads to serious difficulties. Thus we will assume feedback errors resulting in erasure. To prevent deadlock, we assume a timer mechanism is used as in [7]. Moreover, due to the nature of GBN, receiving ACK or NACK for the $i$ th packet means all previous packets have been correctly received.

The GBN protocol is followed similar to, e.g., [8]. The timer is set when a frame is (re)transmitted. If the timer expires before any acknowledgments are received, the frame is retransmitted together with all the succeeding frames. So, the timeout $(T)$ has to be greater than or equal to round trip time $(T \geq k)$. Erroneous ACK/NACK (erasures) are ignored. If an erased feedback were ACK, the message can be implicitly acknowledged by subsequent ACKs or NACKs. If it were a NACK, the transmitter waits until timer expires.

Let us first consider the special case $T=k$. When the acknowledgments are decoded incorrectly, the timer has expired and the message will be retransmitted immediately. Therefore, the discarded ACK or NACK will have the same effect as NACK correctly received. So we have

$$
\begin{aligned}
& \mathbf{M}_{0}=\mathbf{M}_{00} \\
& \mathbf{M}_{1}=\mathbf{M}_{01}+\mathbf{M}_{10}+\mathbf{M}_{11}=\mathbf{M}-\mathbf{M}_{00}
\end{aligned}
$$

We can use (5) to find the throughput in this scenario of $T=k$. Next consider the case $T>k$. Define $d=T-k$ so that $d$ is the maximum number of lost ACK before timer is expired. Then, the transmitter can be represented by a finite state machine with $T+d+1$ states, in state 0 an ACK is correctly decoded. States $L_{1}, L_{2}, \ldots, L_{d}$ correspond to $1,2, \ldots, d$ lost ACKs respectively. Finally in states $W_{j}, j=1,2, \ldots, T$ the transmitter is in the waiting period for timeout or feedback. Let $\mathcal{Z}=\left\{0, L_{1}, L_{2}, \ldots, L_{d}, W_{T}, \ldots, W_{1}\right\}$ represent the set of all states. The block transition probabilities of these states can be given as follows.

For $Z_{t-1}=0, W_{1}$

$$
\operatorname{Pr}\left[Z_{t}=\ell \mid Z_{t-1}\right]= \begin{cases}\mathbf{M}_{00}, & \text { if } \ell=0 \\ \mathbf{M}_{01}, & \text { if } \ell=L_{1} \\ \mathbf{M}_{11}, & \text { if } \ell=W_{T} \\ \mathbf{M}_{10}, & \text { if } \ell=W_{k} \\ \mathbf{0}, & \text { otherwise }\end{cases}
$$

For $Z_{t-1}=L_{1}, L_{2}, \ldots, L_{d-1}$

$$
\operatorname{Pr}\left[Z_{t}=\ell \mid Z_{t-1}=L_{j}\right]= \begin{cases}\mathbf{M}_{00}, & \text { if } \ell=0 \\ \mathbf{M}_{01}, & \text { if } \ell=L_{j+1} \\ \mathbf{M}_{11}, & \text { if } \ell=W_{T-j} \\ \mathbf{M}_{10}, & \text { if } \ell=W_{k} \\ \mathbf{0}, & \text { otherwise }\end{cases}
$$

For $Z_{t-1}=L_{d}$

$$
\operatorname{Pr}\left[Z_{t}=\ell \mid Z_{t-1}=L_{d}\right]= \begin{cases}\mathbf{M}_{00}, & \text { if } \ell=0 \\ \mathbf{M}-\mathbf{M}_{00,}, & \text { if } \ell=W_{k} \\ \mathbf{0}, & \text { otherwise }\end{cases}
$$

For $Z_{t-1}=W_{T}, \ldots, W_{2}$

$$
\operatorname{Pr}\left[Z_{t}=\ell \mid Z_{t-1}=W_{j}\right]= \begin{cases}\mathbf{M}, & \text { if } \ell=W_{j-1} \\ \mathbf{0}, & \text { otherwise }\end{cases}
$$

Let $\mathbf{T}$ be the block transition probability matrix and $\hat{\pi}=$ $\left[\pi_{0} \pi_{L_{1}} \pi_{L_{2}} \ldots \pi_{L_{d}} \pi_{W_{T}} \ldots \pi_{W_{1}}\right]$ be the stationary vectors. Then the stationary vectors can be obtained by solving the following system of equations.

$$
\hat{\pi} \mathbf{T}=\hat{\pi} \text { and } \sum_{\ell \in \mathcal{Z}} \pi_{\ell}=\pi
$$

Where $\pi$ is the stationary probabilities of $\mathbf{M}$ given in (4). The throughput of GBN protocol is given by

$$
\begin{aligned}
\eta & =\left\{\pi_{0}+\left(\pi_{L_{1}}+2 \pi_{L_{2}}+\cdots+d \pi_{L_{d}}\right)\left(\mathbf{M}_{00}+\mathbf{M}_{10}\right)\right\} \mathbf{1} \\
& =\left\{\pi_{0}+\left(\sum_{i=1}^{d} i \pi_{L_{i}}\right)\left(\mathbf{M}_{00}+\mathbf{M}_{10}\right)\right\} \mathbf{1}
\end{aligned}
$$

Let's consider an example of $k=5$ and $T=7$ as in [8].

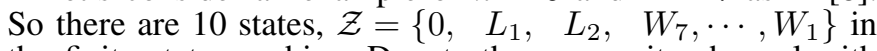
the finite state machine. Denote the composite channel with $\left\{\mathcal{S}, \mathcal{X}, \mathbf{M}_{00}, \mathbf{M}_{01}, \mathbf{M}_{10}, \mathbf{M}_{11}\right\}$. From (6)-(9), the block transition probability matrix will be

$$
\mathbf{T}=\left[\begin{array}{cccccccc}
\mathbf{M}_{00} & \mathbf{M}_{01} & 0 & \mathbf{M}_{11} & 0 & \mathbf{M}_{10} & \cdots & 0 \\
\mathbf{M}_{00} & 0 & \mathbf{M}_{01} & 0 & \mathbf{M}_{11} & \mathbf{M}_{10} & \cdots & 0 \\
\mathbf{M}_{00} & 0 & 0 & 0 & 0 & \mathbf{M}-\mathbf{M}_{00} & \cdots & 0 \\
0 & 0 & 0 & 0 & \mathbf{M} & 0 & \cdots & 0 \\
0 & 0 & 0 & 0 & 0 & \mathbf{M} & \cdots & 0 \\
& & & & & & \ddots & \\
0 & 0 & 0 & 0 & 0 & 0 & \cdots & \mathbf{M} \\
\mathbf{M}_{00} & \mathbf{M}_{01} & 0 & \mathbf{M}_{11} & 0 & \mathbf{M}_{10} & \cdots & 0
\end{array}\right]
$$

The stationary probability vectors are calculated from the following system of equations

$$
\begin{aligned}
\pi_{0} & =\left(\pi_{0}+\pi_{L_{1}}+\pi_{L_{2}}+\pi_{W_{1}}\right) \mathbf{M}_{00} \\
\pi_{L_{1}} & =\left(\pi_{0}+\pi_{W_{1}}\right) \mathbf{M}_{01} \\
\pi_{L_{2}} & =\pi_{L_{1}} \mathbf{M}_{01} \\
\pi_{W_{7}} & =\left(\pi_{0}+\pi_{W_{1}}\right) \mathbf{M}_{11} \\
\pi_{W_{6}} & =\pi_{L_{1}} \mathbf{M}_{11}+\pi_{W_{7}} \mathbf{M} \\
\pi_{W_{5}} & =\left(\pi_{0}+\pi_{L_{1}}+\pi_{W_{1}}\right) \mathbf{M}_{10}+\pi_{L_{2}}\left(\mathbf{M}-\mathbf{M}_{00}\right) \\
\quad & \quad+\pi_{W_{6}} \mathbf{M} \\
\pi_{W_{j}} & =\pi_{W_{j+1}} \mathbf{M}, \quad j=4,3,2 \text { and } 1 \\
\sum_{\ell \in \mathcal{Z}} \pi_{\ell} & =\pi
\end{aligned}
$$


Substitute $\mu=\pi_{0}+\pi_{W_{1}}$. After some algebra, the following equation yields $\mu$.

$$
\begin{aligned}
\mu\left\{\mathbf{I}+\mathbf{M}_{01}+\mathbf{M}_{01}^{2}+\mathbf{M}_{11}+\mathbf{M}_{01} \mathbf{M}_{11}+\mathbf{M}_{11} \mathbf{M}\right. \\
+\left[\mathbf{M}_{10}+\mathbf{M}_{01} \mathbf{M}_{10}+\mathbf{M}_{01}^{2} \mathbf{M}-\mathbf{M}_{01}^{2} \mathbf{M}_{00}\right. \\
\left.\left.+\mathbf{M}_{01} \mathbf{M}_{11} \mathbf{M}+\mathbf{M}_{11} \mathbf{M} \mathbf{M}\right] \mathbf{Q}(k)\right\}=\pi
\end{aligned}
$$

Where $\mathbf{I}$ is an identity matrix and $\mathbf{Q}(k)=\sum_{i=0}^{k-2} \mathbf{M}^{i}$. From the first three equations of system (12), we have

$$
\begin{aligned}
\pi_{0} & =\mu\left(\mathbf{M}_{00}+\mathbf{M}_{01} \mathbf{M}_{00}+\mathbf{M}_{01} \mathbf{M}_{01} \mathbf{M}_{00}\right) \\
\pi_{L_{1}} & =\mu \mathbf{M}_{01} \\
\pi_{L_{2}} & =\mu \mathbf{M}_{01} \mathbf{M}_{01}
\end{aligned}
$$

Finally substituting $\pi_{0}, \pi_{L_{1}}$ and $\pi_{L_{2}}$ into (11) will give the throughput of GBN protocol.

\section{RElationship With Renewal Reward Theory}

The technique of renewal reward processes [13] has been used in [8] for the analysis of Go-Back-N in simple two-state Markovian channels with unreliable feedback. In this section, we examine the relationship of our analysis to the renewal reward method. Our methodology is, in a sense, equivalent to a generalization of renewal reward methods from scalar to matrix form.

Denote by $R(\tau)$ the number of correct receptions up to a time $\tau$, which in our case is the reward. From a fundamental theorem of the renewal reward processes, we have

$$
\lim _{\tau \rightarrow \infty} \frac{R(\tau)}{\tau}=\frac{E[R]}{E[D]}
$$

where $E[D]$ is the average cycle duration from one state to the next. In [8], the calculations for a Markovian channel are made by calculating reward and delay transition matrices.

In our case, we are analyzing a nonstationary block-fading channel, therefore using the renewal reward method in the manner of [8] is not straight forward. Using our previous developments, we note that our transition probabilities are calculated for one transition per time interval, therefore in our case $E[D]=1$. We can now calculate the average reward using the earlier developments. We demonstrate the calculations for the special case of $T=k$. Using the same notation as before, let $\mathcal{Z}=\left\{0, W_{T}, \ldots, W_{1}\right\}$ represent the set of all states. Then:

$$
\mathbf{T}_{(k+1) N \times(k+1) N}=\left[\begin{array}{cccccc}
\mathbf{M}_{0} & \mathbf{M}_{1} & 0 & 0 & \ldots & 0 \\
0 & 0 & \mathbf{M} & 0 & \ldots & 0 \\
0 & 0 & 0 & \mathbf{M} & \ldots & 0 \\
\vdots & & & & \ddots & \vdots \\
0 & 0 & 0 & 0 & \ldots & \mathbf{M} \\
\mathbf{M}_{0} & \mathbf{M}_{1} & 0 & 0 & \ldots & 0
\end{array}\right]
$$

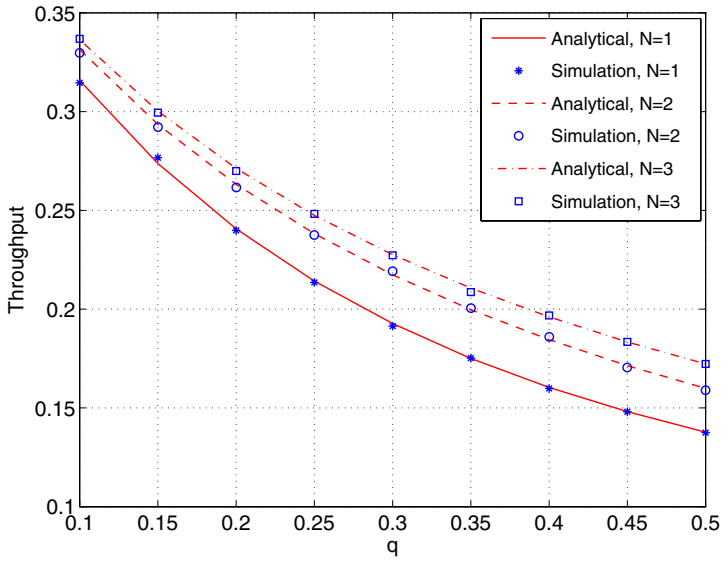

Fig. 2. Throughput, $\eta$, vs. $q$ :analytical and simulation results for $r=0.3$, $\varepsilon_{G}=0.2, \varepsilon_{B}=0.8$ and $N=1,2,3$.

Now we construct the reward matrix.

$\mathbf{R}_{(k+1) N \times(k+1) N}=\left[\begin{array}{ccccc}1 & 0 & 0 & \ldots & 0 \\ 1 & 0 & 0 & \ldots & 0 \\ \vdots & & & \ddots & \vdots \\ 1 & 0 & 0 & \ldots & 0\end{array}\right] \otimes\left[\begin{array}{ccc}1 & \ldots & 1 \\ \vdots & & \vdots \\ 1 & \ldots & 1\end{array}\right]_{N \times N}$

where $\otimes$ denotes the Kronecker product, and the matrix $\mathbf{R}$ essentially says that a unit reward is collected whenever a correct acknowledgement is received. The average reward can be calculated as:

$$
E[R]=\sum_{i}\left(\mathbf{T ~ R}^{t}\right)_{i i} \hat{\pi}_{i}
$$

where $\hat{\pi}=\left[\pi_{0} \pi_{W_{T}} \ldots \pi_{W_{1}}\right]$ is the stationary vector of the transition matrix $\mathbf{T}$. In the case of $T=k$, the average reward expression can be collected into matrix form:

$$
E[R]=\left(\pi_{0}+\pi_{W_{1}}\right) \mathbf{M}_{0} \mathbf{1}
$$

One may wish to verify that this is consistent with the expression obtained using the previous method. To do so, using the system of equations (10), we can show that

$$
\left(\pi_{0}+\pi_{W_{1}}\right) \mathbf{M}_{0} \mathbf{1}=\pi_{0} \mathbf{1}=\pi\left[\mathbf{I}-\mathbf{M}_{1} \mathbf{Q}(k)\right]^{-1} \mathbf{M}_{0} \mathbf{1}
$$

\section{Numerical Results}

We consider a channel with two quality levels, whose transition matrix is given in (3). Recall that the parameters of the forward channel are error probabilities $\varepsilon_{G}, \varepsilon_{B}$, state transition probabilities $r, q$, round-trip time $k$ and length of block fading $N$. The round-trip time indicates the distance of the transmitter and receiver, as well as their processing delay; for our simulations we set $k=5$. The block fading length is an indicator of channel variations, which in part depends on user mobility as well as the dynamics of the environment. Fig. 2 shows the throughput vs $q$ under block fading channel for $N=1,2$, and 3 . The throughput under block fading channels is higher than i.i.d. channels when $r+q<1$, a result that has been reported in [5].

Fig. 3 shows that throughput initially improves with length of block fading, but it quickly saturates as $N \rightarrow \infty$. The behavior can be explained as follows: Note that the average 


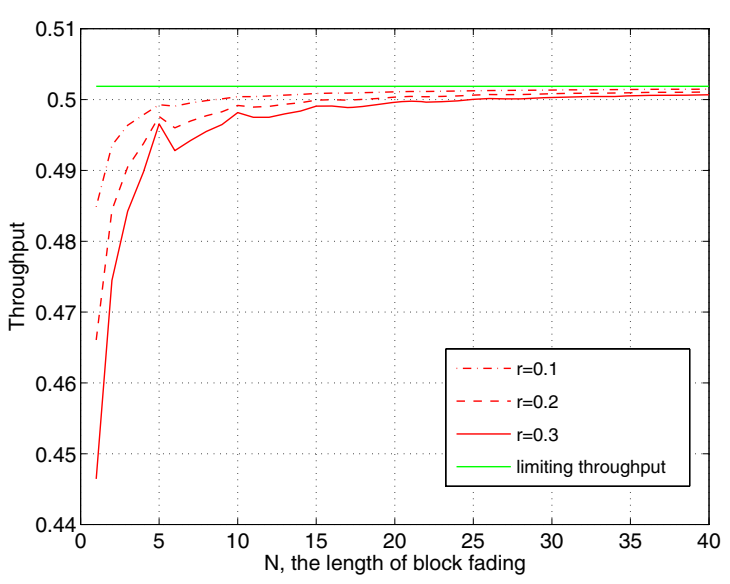

Fig. 3. Throughput, $\eta$, vs. length of block fading, $N$ for $\varepsilon_{G}=0.1, \varepsilon_{B}=$ 0.7 , block error rate $=0.25$ and $r=0.1,0.2,0.3$.

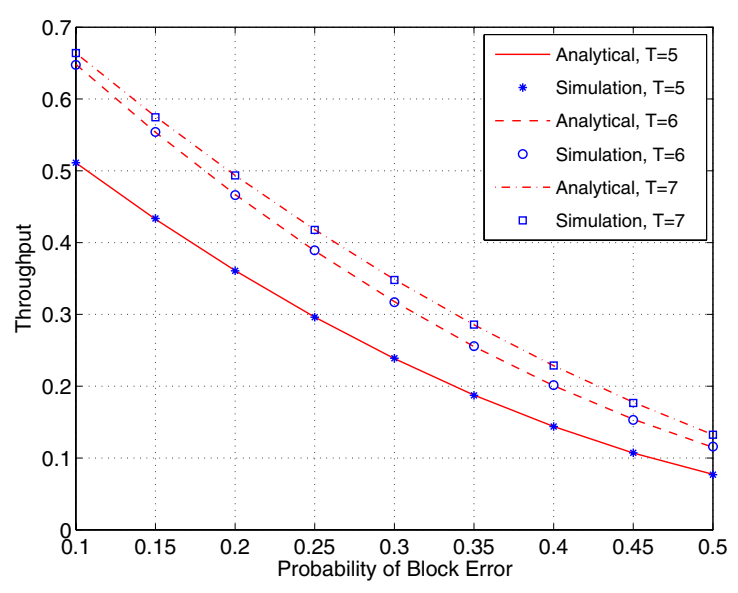

Fig. 4. Throughput vs block error rate for $N=3, r=0.3, \varepsilon_{G}=0.07$, $\varepsilon_{B}=0.7, k=5$ and $T=5,6,7$.

number of losses is held constant in this analysis. As we increase $N$, the channel block length, the increased memory of the channel means that losses are more clustered, which is good for the GBN protocol, since each time an error is observed, several packets are discarded anyway. In this example, each time an error is observed, 5 packets must be retransmitted, so we see that increased throughput levels off around $N=5$.

In this figure we also observe another interesting phenomenon: there are small jumps in throughput when $N$ is a multiple of $T$. This can be explained as follows: If $N=T$, when a block is in deep fade, one may recover by exactly one round of retransmission, without any waste (without GBN retransmitting packets that were correctly received). If $N=2 T$, one may recover with exactly two rounds of retransmission without any waste, etc.

Under block fading with unreliable feedback, the throughput is shown in Fig. 4 when the reverse channel has the same statistics as the forward channel, i.e., $\left\{\mathcal{S}^{(f)}, \mathcal{X}^{(f)}, \mathbf{M}_{1}^{(f)}, \mathbf{M}_{0}^{(f)}\right\}=\left\{\mathcal{S}^{(b)}, \mathcal{X}^{(b)}, \mathbf{M}_{0}^{(b)}, \mathbf{M}_{1}^{(b)}\right\}$. We then investigate the asymmetric forward/backward channel by fixing $B E R=0.1$ in backward channel and varying BER in the forward channel; the results appear in Fig. 5.

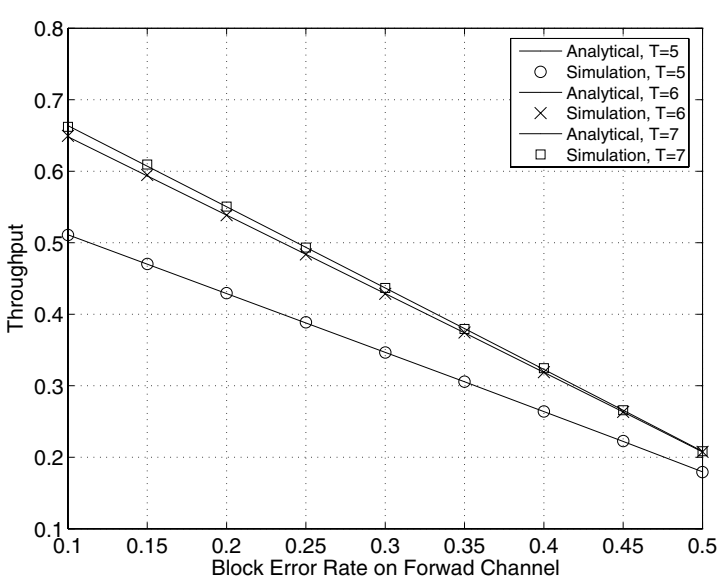

Fig. 5. Throughput for asymmetric forward/backward channels. The backward channel is fixed at $\mathrm{BER}=0.1$ and the forward channel is allowed to vary. Other parameters are $N=3, r=0.3, \varepsilon_{G}=0.07, \varepsilon_{B}=0.7, k=5$ and $T=5,6,7$.

\section{CONCLUSION}

In this paper, we study Go-Back-N ARQ under block fading. By characterizing the block fading with a hidden Markov model, we calculate the throughput of GBN with both reliable and unreliable feedback. Feedback errors are modeled by erasure which normally occur in practical data communications. Future research directions may include analysis of the delay time of GBN protocol as well as modified GBN protocols such as GBN with stutter or GBN with more than one NACK.

\section{REFERENCES}

[1] H. S. Wang and N. Moayeri, "Finite-state Markov channel-A useful model for radio communication channels," IEEE Trans. Veh. Technol., vol. 44, no. 1, pp. 163-171, Feb. 1995.

[2] H. S. Wang and P.-C. Chang, "On verifying the first-order Markovian assumption for a Rayleigh fading channel model," IEEE Trans. Veh. Technol., vol. 45, no. 2, pp. 353-357, May 1996.

[3] Q. Zhang and S. A. Kassam, "Finite-state Markov model for Rayleigh fading channels," IEEE Trans. Commun., vol. 47, no. 11, pp. 1688-1692, Nov. 1999.

[4] P. Bergamo, D. Maniezzo, A. Giovanardi, G. Mazzini, and M. Zorzi, "An improved Markov chain description for fading processes," in Proc. IEEE ICC, Apr.-May 2002, pp. 1347-1351.

[5] C. H. C. Leung, Y. Kikumoto, and S. A. Sorensen, "The throughput efficiency of the Go-Back-N ARQ scheme under Markov and related error structures," IEEE Trans. Commun., vol. 36, pp. 231-234, Feb. 1988.

[6] D. L. Lu and J. F. Chang, "Performance of ARQ protocols in nonindependent channel errors," IEEE Trans. Commun., vol. 41, no. 5, pp. 721-730, May 1993.

[7] Y. J. Cho and C. K. Un, "Performance analysis of ARQ error controls under Markovian block error pattern," IEEE Trans. Commun., vol. 42, pp. 2051-2061, Feb.-Apr. 1994.

[8] M. Zorzi and R. R. Rao, "Throughput analysis of Go-Back-N ARQ in Markov channels with unreliable feedback," in Proc. IEEE ICC, June 1995, pp. 1232-1237.

[9] W. Turin, "Throughput analysis of the Go-Back-N protocol in fading radio channels," IEEE J. Select. Areas Commun., vol. 17, no. 5, pp. 881-887, May 1999.

[10] R. J. McEliece and W. E. Stark, "Channels with block interference," IEEE Trans. Inform. Theory, vol. 30, no. 1, pp. 44-53, Jan. 1984.

[11] R. A. Howard, Dynamic Probabilistic Systems. New York: John Wiley \& Sons, Inc., 1971.

[12] W. Turin, Performance Analysis and Modeling of Digital Transmission Systems. New York: Kluwer Academic/Plenum Publishers, 2004.

[13] S. Ross, Random Processes. New York: John Wiley \& Sons, Inc., 1983. 